\title{
La violenza domestica assistita
}

\author{
Vincenzo M. Mastronardi, ${ }^{1}$ Simone Montaldo, ${ }^{2}$ Ester Deplano ${ }^{3}$ \\ ${ }^{1}$ Psichiatra già Direttore della Cattedra di Psicopatologia forense Università di Roma Sapienza; Direttore del Master di \\ Criminologia, Scienze investigative e Strategiche per la Sicurezza Unitelma Sapienza; Docente del Corso di Laurea \\ Magistrale in Investigazione, Criminalità e Sicurezza Internazionale UNINT (Università degli Studi Internazionali di \\ Roma); ${ }^{2}$ Dottore in Psicologia clinica; Criminologo; Docente di Psicologia della testimonianza presso il Master di I livello \\ in Criminologia e Scienze strategiche della Sapienza Università di Roma; Membro del BAHID (British Association for \\ Human Identification); ${ }^{3}$ Avvocato, Criminologa, Grafologa forense, Italia
}

Il problema della violenza domestica ha assunto oggi grande rilevanza, sia nel campo della ricerca scientifica che nel campo delle politiche sociali, dell'azione penale e della tutela legale delle vittime. Purtroppo, bisogna rilevare che la grande attenzione mediatica concentratasi sui casi di violenza domestica, ha prodotto una serie di errate convinzioni e luoghi comuni, che rischiano di affliggere tanto il giudizio comune quanto quello legale, assai più delicato per le implicazioni dirette e indirette sui soggetti coinvolti. Una scarsa chiarezza sulle caratteristiche del fenomeno incide su tutte le fasi e le tipologie di valutazione deputate all'accertamento dei casi specifici e, per naturale estensione, anche sui programmi di prevenzione e sostegno, dedicati in particolare alle vittime.

Una delle forme di maggiore complessità in tema di Violenza nelle relazioni intime (Intimate Partner Violence IPV) è rappresentata dalle circostanze in cui la violenza domestica è agita in contesti familiari dove sono presenti dei figli (specie se piccoli), che assistono agli abusi, pur senza esserne oggetto. Ebbene esiste un ampio corpo di ricerche che evidenziano che, in simili circostanze, la vittimizzazione da violenza assistita (Witnessing domestic violence, WDV) può essere altrettanto consistente

Correspondence: Vincenzo M. Mastronardi.

E-mail: vincenzo.mastronardi@gmail.com

Received for publication: 7 January 2018.

Revision received: 6 April 2018.

Accepted for publication: 10 May 2018.

This article is distributed under the terms of the Creative Commons Attribution Noncommercial License (by-nc 4.0) which permits any noncommercial use, distribution, and reproduction in any medium, provided the original author(s) and source are credited.

(C) Copyright V.M. Mastronardi et al., 2018

Licensee PAGEPress, Italy

Rivista di Psicopatologia Forense, Medicina Legale, Criminologia 2018; $23: 37$

doi:10.4081/psyco.2018.37 quanto quella da violenza subita, con drammatici effetti negativi a breve, medio e lungo termine.

Le esperienze negative vissute in età infantile (ACEs, Adverse Childhood experiences) possono determinare un potente fattore di rischio per lo sviluppo di patologie psichiche, fisiche, disturbi dell'adattamento e anche problemi comportamentali di notevole gravità (come ad esempio assumere, una volta in età adulta, il ruolo di perpetratori in contesti di Violenza nelle relazioni intime).

La categoria delle esperienze negative infantili comprende un ampio range di possibili eventi (Anda et al., 2010; Centers for Disease Control and Prevention -CDC-, 2010), fra cui:

- Avere subito abusi emotivi, fisici o sessuali

- Avere assistito a violenze fra i genitori

- Avere avuto genitori con patologie mentali

- Avere avuto genitori incarcerati

- Avere avuto genitori con problemi di abuso di sostanze

Come si nota, l'essere stati anche "semplici" testimoni di violenze in famiglia, rappresenta una tipologia di evento dalla forte connotazione emotivamente negativa, assimilabile a gravi forme di abuso diretto.

La ricerca mostra come la violenza assistita possa configurarsi come un notevole fattore di rischio nello sviluppo psico-fisico del minore e come, in quanto tale, meriti una particolare attenzione nel contesto della prevenzione e della terapia dei soggetti che hanno esperito simili eventi. Ne deriva che "il ruolo di vittima", nei casi di violenza domestica, non è attribuibile solo a chi subisce le azioni violente (uno dei partner), ma anche a chi (come i figli), pur non essendo oggetto di tali violenze, ne è coinvolto come testimone/osservatore.

Alcune recenti ricerche hanno dunque stimato che, nella popolazione generale, 6 persone su dieci sono state esposte nel corso della vita, ad almeno un evento di tipo ACE (Brown et al., 2013a), e altri studi, hanno mostrato una correlazione fra gli ACEs ed effetti negativi sulla salute (sia fisica che psichica), come riportato nella Tabella 1. 
Tabella 1. Effetti negativi degli ACEs sulla salute (a lungo termine).

\begin{tabular}{|c|c|}
\hline \multicolumn{2}{|l|}{ Patologie organiche } \\
\hline Cancro & Brown et al., 2013a, 2010 \\
\hline Malattie cardiovascolari & Felitti et al., 1998; Dong et al., 2004 \\
\hline Diabete & Felitti et al., 1998 \\
\hline \multicolumn{2}{|l|}{ Patologie mentali } \\
\hline Abuso di sostanze & $\begin{array}{l}\text { Felitti et al., 1998; Jewkes et al., 2010; } \\
\text { Oladeji et al., 2010; Strine et al., 2012; } \\
\text { Wu et al., } 2010\end{array}$ \\
\hline Depressione & $\begin{array}{l}\text { Felitti et al., 1998; Jewkes et al., 2010; } \\
\text { Chapman et al., 2004; Pickles et al., } \\
\text { 2010; Sareen et al., 2013; Nicodimos, } \\
\text { Gelaye, Williams, Berhane, } 2009\end{array}$ \\
\hline Ansia & Sareen et al., 2013 \\
\hline PTSD (Posttraumatic stre & $\begin{array}{l}\text { ss disorder) } \\
\text { Wolfe et al., 1994; Luthra, Abramovitz, } \\
\text { Greenberg, Schoor, Newcorn, et al., } \\
\text { 2009; Silva, Alpert, Munoz, Singh, } \\
\text { Matzner, et al., } 2000\end{array}$ \\
\hline Comportamento violento & $\begin{array}{l}\text { nelle relazioni intime in età adulta } \\
\text { McKinney et al., } 2009\end{array}$ \\
\hline
\end{tabular}

Come già accennato tali indicazioni della ricerca conducono alla possibilità di una costante revisione dei programmi di prevenzione e trattamento. L'osservazione sugli effetti della violenza assistita ha ormai assunto una dimensione temporale di tipo lifespan (che riguarda l'intero corso della vita) e che consente una migliore capacità diagnostica, una più ampia e approfondita conoscenza sull'eziologia di alcuni disturbi psicologici e comportamentali e una migliore efficacia prognostica sul progresso e sull'evoluzione dei disturbi correlati alla violenza assistita. Una visione così ampliata sul fenomeno, fa si che si possa tenere conto delle esperienze negative in infanzia (ad es. violenza assistita) non solo nel trattamento delle vittime, ma anche in quello dei perpetratori, che spesso hanno, nella propria storia di vita, vicende di tipo ACEs, che assumono un ruolo decisivo nel corso dello sviluppo e nell'evoluzione di determinati quadri clinici e comportamentali.

Dalla ricerca emerge un progresso temporalmente lineare degli effetti negativi a lungo termine degli ACEs, che individua le conseguenze psicologiche in età adulta (ad es. PTSD, Depressione e Abuso di sostanze) come mediatori, in un'associazione indiretta, fra eventi negativi nell'infanzia (come la violenza assistita) e lo sviluppo di comportamenti abusanti nelle relazioni intime in età adulta.

Brown e colleghi (2015) hanno fornito un'efficace rappresentazione grafica di questo fenomeno nel loro Mediational model, nel quale descrivono l'associazione indiretta fra le esperienze negative nell'infanzia (ACEs) e i comportamenti di violenza nelle relazioni intime in età adulta.

Si evidenzia inoltre che, in particolare, l'aver subito abusi o l'essere stati testimoni di violenze fra i genitori in età infantile, sono fattori di rischio indipendenti rispetto all'agire comportamenti violenti/aggressioni nelle relazioni intime in età adulta (Ehrensaft et al., 2003).

Una individuazione precoce di situazioni di vittimiz- zazione da violenza assistita in minori che vivono in contesti dove è presente violenza domestica fra i genitori, consentirà un intervento tempestivo, atto a prevenire conseguenze negative per la salute fisica e soprattutto psichica, con una maggiore possibilità di prevenire $e$ minimizzare l'insorgere e l'aggravarsi (nel corso dello sviluppo) di tali effetti. Tale prevenzione, nella logica del rapporto fra $\mathrm{ACEs}$ e violenza agita in età adulta, potrà avere un effetto anche sull'insorgenza di futuri comportamenti abusanti.

\section{Gli effetti negativi della violenza assistita nell'infanzia, nei giovani adulti: dallo sviluppo della morfologia cerebrale, ai disturbi psichici e comportamentali}

L'attuale scienza della mente consente di ampliare lo studio del funzionamento fisiologico e di quello patologico, in una prospettiva ampliata, che integra lo studio delle funzioni cognitive e psichiche con le conoscenze in campo neurofisiologico e della biologia molecolare, adeguando la capacità descrittiva dell'osservazione alla complessità delle funzioni mentali. E' dunque chiaro che, se si vuole approfondire la conoscenza di condizioni psicopatologiche connesse ad esperienze traumatiche vissute nell'infanzia, si deve fare riferimento non solo alle componenti psico-affettive dello sviluppo, ma anche a quelle legate allo sviluppo morfologico delle strutture cerebrali sottostanti alle funzioni adattive, sia sul piano cognitivo che su quello esecutivo.

E' noto che $\mathrm{i}$ bambini che si trovano a vivere in contesti familiari dove si verificano violenze fra i genitori, si trovano comunemente a vedere, sentire e intervenire in episodi di violenza/aggressione (Fantuzzo, Boruch, Beriama, Atkins, Marcus, 1997).

Abbiamo visto come la violenza assistita nell'infanzia, determini negli individui potenziali effetti negativi (quali ad es. PTSD, depressione etc.), che progrediscono per forma e gravità attraverso le diverse fasi dello sviluppo. Nel novero dei possibili effetti negativi rientra anche una riduzione delle capacità cognitive (Tomoda, Polcari, Anderson, Teicher, 2012), con tutto ciò che questo comporta sullo sviluppo delle capacità adattive e di coping sociale.

Le esperienze negative nel corso dell'infanzia possono influire direttamente sulla morfologia cerebrale e sull'integrità e lo sviluppo di specifiche aree. I ricercatori hanno individuato, ad esempio, una riduzione nell'integrità del fascicolo longitudinale inferiore (ILF), che connette la corteccia visiva al sistema limbico, in un campione di soggetti che avevano assistito a violenze fra i genitori nel corso dell'infanzia (Choi, Jeong, Polcari, Rohan, Teicher, 2012).

La ricerca ha approfondito ulteriormente lo studio degli effetti fisici della violenza assistita, fino ad ampliare l'osservazione al campo dello sviluppo strutturale di aree cerebrali di grande importanza funzionale, quale, ad esempio, il volume della materia grigia della corteccia (GMV, Gray Matter 
Volume) e lo spessore corticale (cortical tichness). La ricerca (Tomoda, Polcari, Anderson, Teicher, 2012) ha mostrato che i soggetti che hanno avuto esperienze di Violenza domestica assistita, mostrano, rispetto ai soggetti di controllo, una riduzione di circa il $6,1 \%$ del Volume della materia grigia nel Giro linguale di destra ${ }^{1}$ e anche una riduzione nello spessore di quest'area. Tale riduzione di spessore è stata osservata parallelamente in area V2 bilaterale e nel polo occipitale sinistro. Queste regioni cerebrali sono particolarmente esposte agli effetti della violenza assistita fra gli $11 \mathrm{e}$ i 13 anni di età. Un dato di particolare interesse è costituito dal fatto che simili effetti sul volume e sullo spessore delle medesime aree cerebrali sono stati osservati osservato in soggetti che erano stati vittime di abusi sessuali, dove la corteccia visiva appariva come quella primariamente danneggiata dall'esperienza. In sintesi, le regioni cerebrali che processano e trasmettono gli input sensoriali negativi dell'abuso possono essere specificamente modificate dall'esperienza, specie in soggetti esposti ad un singolo e specifico tipo di maltrattamento. L'esposizione a diverse forme di maltrattamento è più comunemente associata ad alterazioni morfologiche nelle regioni corticolimbiche. Tali osservazioni si adattano agli studi preclinici che individuano la corteccia visiva come un'area cerebrale dotata di notevole plasticità.

Queste evidenze mostrano in modo chiaro e incontrovertibile, quanto la violenza assistita possa condizionare le vittime nel loro sviluppo fisico e psicologico, in modo grave e pervasivo e con un progresso esponenziale del danno correlato.

\section{Conclusioni}

Dalla breve overview sopra sviluppata sul tema della Violenza assistita e sugli effetti negativi che questa ha nel breve, medio e lungo termine sui soggetti che ne sono stati vittime nel corso dell'infanzia, risulta evidente quanto sia importante porre una grande attenzione su questa specifica forma di vittimizzazione. Nei casi di violenza domestica, ogni intervento (sia di tipo legale che di predisposizione di specifiche politiche sociali e sanitarie di prevenzione e trattamento) dovrà tenere presenti alcuni punti chiave, che sono:

- Le vittime della violenza domestica non sono soltanto quelle che sono direttamente oggetto di aggressione (uno dei partner), ma (quando presenti nel contesto familiare), anche i figli che sono testimoni di tale violenze sono potenzialmente esposti a gravi danni fisici e psicologici.

- I potenziali danni della violenza assistita vanno da

1 Il giro linguale è una struttura a forma di lingua che si trova sull'aspetto mediale del lobo occipitale lungo la superficie infero-cavernosa (tentoriale). Quest'area è associata ad una varietà di funzioni visive, come, ad esempio, il riconoscimento dei volti. disfunzioni nello sviluppo morfologico cerebrale, fino a disturbi di tipo organico, psicologico e comportamentale.

- Gli effetti negativi a medio e lungo termine della violenza assistita, sono assimilabili per gravità e pervasività a quelli prodotti dalla violenza subita.

- Uno dei possibili esiti a lungo termine della violenza assistita in infanzia è quello di agire violenza nelle relazioni intime, in età adulta.

- La violenza assistita costituisce un fattore di rischio indipendente rispetto alla probabilità di sviluppare comportamenti abusanti in età adulta.

- Gli interventi di prevenzione e trattamento dovrebbero essere rivolti a tutti i soggetti coinvolti in contesti violenti e abusanti, ovvero: i partner che subiscono la violenza, $i$ partner che agiscono la violenza e $i$ figli che assistono alla violenza.

- Interventi tempestivi sui soggetti che sono vittime di violenza assistita possono avere maggiore efficacia nella minimizzazione dei fattori di rischio sugli effetti negativi a medio e lungo termine.

\section{Bibliografia}

Anda RF, Butchart A, Felitti VJ, Brown DW. (2010). Building a framework for global surveillance of the public health implications of adverse childhood experiences. Am. J. Prev. Med.; 39:93-98. [PubMed: 20547282].

Brown DW, Anda RF, Felitti VJ, et al. (2010). Adverse childhood experiences are associated with the risk of lung cancer: a prospective cohort study. BMC Public Health; 10:20. [PubMed: 20085623]

Brown MJ, Perera RA, Masho SW, Mezuk B, and Cohen SA. (2015). Adverse childhood experiences and intimate partner aggression in the US: Sex differences and similarities in psychosocial mediation. Soc Sci Med 131:4857. doi:10.1016/j.socscimed.2015.02.044.

Brown MJ, Thacker LR, Cohen SA. (2013). Association between adverse childhood experiences and diagnosis of cancer. PLoS One.; 8:e65524. [PubMed: 23776494].

Centers for Disease Control Prevention (CDC). (2010). Adverse Childhood Experiences Reported by Adults Five States, 2009. MMWR Morb Mortal Wkly Rep.; 59:1609-1613. [PubMed: 21160456].

Chapman DP, Whitfield CL, Felitti VJ, Dube SR, Edwards VJ, Anda RF. (2004). Adverse childhood experiences and the risk of depressive disorders in adulthood. J. Affect Disord.; 82:217-225. [PubMed: 15488250].

Choi J, Jeong B, Polcari A, Rohan ML, Teicher MH. (2012). Reduced fractional anisotropy in the visual limbic pathway of young adults witnessing domestic violence in childhood. Neuroimage 59: 1071-1079.

Dong M, Giles WH, Felitti VJ, et al. (2004). Insights into causal pathways for ischemic heart disease: adverse childhood experiences study. Circulation; 110:17611766. [PubMed: 15381652]. 
Ehrensaft MK, Cohen P, Brown J, Smailes E, Chen H, Johnson JG. (2003). Intergenerational transmission of partner violence: a 20-year prospective study. J. Consult Clin. Psychol.; 71:741-753. [PubMed: 12924679].

Fantuzzo J, Boruch R, Beriama A, Atkins M, Marcus S. (1997). Domestic violence and children: prevalence and risk in five major U.S. cities. J Am Acad Child Adolesc Psychiatry 36: 116-122.

Felitti VJ, Anda RF, Nordenberg D, et al. (1998). Relationship of childhood abuse and household dysfunction to many of the leading causes of death in adults. The Adverse Childhood Experiences (ACE) Study. Am. J. Prev. Med; 14:245-258. [PubMed: 9635069].

Jewkes RK, Dunkle K, Nduna M, Jama PN, Puren A. (2010). Associations between childhood adversity and depression, substance abuse and HIV and HSV2 incident infections in rural South African youth. Child. Abuse Negl.; 34:833-841. [PubMed: 20943270].

Luthra R, Abramovitz R, Greenberg R, Schoor A, Newcorn J, et al. (2009). Relationship between type of trauma exposure and posttraumatic stress disorder among urban children and adolescents. J Interpers Violence 24: 1919-1927.

McKinney CM, Caetano R, Ramisetty-Mikler S, Nelson S. (2009). Childhood family violence and perpetration and victimization of intimate partner violence: findings from a national population-based study of couples. Ann. Epidemiol.; 19:25-32. [PubMed: 18835525].

Nicodimos S, Gelaye BS, Williams MA, Berhane Y. (2009). Associations between witnessing parental violence and experiencing symptoms of depression among college students. East Afr J Public Health 6: 184-190.

Oladeji BD, Makanjuola VA, Gureje O. (2010). Family- related adverse childhood experiences as risk factors for psychiatric disorders in Nigeria. Br. J. Psychiatry; 196:186-191. [PubMed: 20194539].

Pickles A, Aglan A, Collishaw S, Messer J, Rutter M, Maughan B. (2010). Predictors of suicidality across the life span: the Isle of Wight study. Psychol. Med.; 40:1453-1466. [PubMed: 19939326].

Sareen J, Henriksen CA, Bolton SL, Afifi TO, Stein MB, Asmundson GJ. (2013). Adverse childhood experiences in relation to mood and anxiety disorders in a population-based sample of active military personnel. Psychol. Med.; 43:73-84. [PubMed: 22608015].

Silva RR, Alpert M, Munoz DM, Singh S, Matzner F, et al. (2000). Stress and vulnerability to posttraumatic stress disorder in children and adolescents. Am J Psychiatry 157: 1229-1235.

Strine TW, Dube SR, Edwards VJ, et al. (2012). Associations between adverse childhood experiences, psychological distress, and adult alcohol problems. Am. J. Health Behav.; 36:408-423. [PubMed: 22370441].

Tomoda A, Polcari A, Anderson CM, Teicher MH. (2012). Reduced Visual Cortex Gray Matter Volume and Thickness in Young Adults Who Witnessed Domestic Violence during Childhood. PLoS ONE 7(12): e52528. doi:10.1371/journal.pone.0052528.

Wolfe DA, Sas L, Wekerle C. (1994). Factors associated with the development of posttraumatic stress disorder among child victims of sexual abuse. Child. Abuse Negl.; 18:37-50. [PubMed: 8124597].

Wu NS, Schairer LC, Dellor E, Grella C. (2010). Childhood trauma and health outcomes in adults with comorbid substance abuse and mental health disorders. Addict. Behav; 35:68-71. [PubMed: 19775820].

\section{Ricerca giurisprudenziale a cura di Ester Deplano}

\section{Prima sentenza}

Trib. Piacenza Dec., 23/10/2008

Pagani c. Poggi COMPETENZA E GIURISDIZIONE PENALE

Competenza, in genere

\section{MALTRATTAMENTI IN FAMIGLIA}

Nelle fattispecie di c.d. violenza assistita, ove la vittima diretta dei maltrattamenti è un genitore e i figli vengono loro malgrado costretti ad assistervi, sussiste una sovrapposizione di competenze tra il giudice civile, adito ai sensi degli artt. 342-bis e 342-ter c.c. e dell'art. 736-bis c.p.c., e il tribunale per i minorenni. Tale sovrapposizione di competenze non preclude al giudice civile di pronunciare - intervenuto decreto del tribunale per i minorenni che dispone, ai sensi degli artt. 333 e 336 c.c., l'allontanamento del genitore violento dalla casa familiare e l'affidamento del figlio minore - non solo l'allontanamento dalla casa familiare del medesimo genitore, ma anche la cessazione della condotta pregiudizievole, quale contenuto essenziale dell'ordine di protezione di cui agli artt. 342-bis e 342-ter c.c..

\section{Seconda sentenza}

\section{SUPREMA CORTE DI CASSAZIONE SEZIONE VI PENALE}

Sentenza 29 gennaio 2015 n. 4332

Presidente Milo - Relatore Lanza

Nella motivazione della sentenza, si richiama l'orientamento per il quale integrano il delitto di cui all'art. 572 c.p. 
non solo fatti commissivi, sistematicamente lesivi della personalità della persona offesa, ma anche quelle condotte omissive connotate da una deliberata indifferenza e trascuratezza verso gli elementari bisogni affettivi ed esistenziali della "persona debole" da tutelare.

\section{Ritenuto in fatto}

1. Il P.M. presso il Tribunale di Roma ricorre avverso l'ordinanza 17 giugno 2014 del Tribunale della libertà di Roma, pronunciata nei confronti di T.E. , nella parte in cui il provvedimento cautelare è stato annullato con riferimento ai contestati maltrattamenti nei confronti dei figli.

2. Il Tribunale del riesame, nel confermare i gravi indizi di colpevolezza quanto al delitto contestato in danno della moglie del ricorrente, ha invece annullato l'ordinanza cautelare, quanto ai contestati maltrattamenti nei confronti dei figli, rilevando che gli elementi raccolti, mettono in luce soltanto pochi episodi isolati in cui figurano i figli minori del T. (quello della doccia, quello dello schiaffo indirizzato alla $S$. che colpiva anche il bambino in braccio, quello recente del livido al braccio di $M$. , peraltro spiegato dal T. quale conseguenza non di Violenza gratuita bensi del tentativo di trattenere il figlio che aveva aperto lo sportello dell'auto già in movimento), episodi definiti “comunque senz'altro inidonei a disegnare la condotta continua e sistematica richiesta dalla norma in addebito per l'integrazione della fattispecie".

3. Il provvedimento peraltro ha preso atto della giurisprudenza (idest: cass. pen. sezione. 5, 41142/2010) che ritiene configurabile il reato di cui all'art. 572 c.p. in danno dei figli per episodi di violenza in danno della convivente, in ragione delle ricadute del comportamento del genitore violento sui minori, rilevando che in quel caso - ben diverso per gravità ed esiti - i figli avevano timore persino di andare a scuola perché ciò avrebbe loro impedito di difendere adeguatamente la propria madre in ragione degli atti vessatori cui avevano assistito ad opera del padre.

4. Per il Tribunale invece, nel caso di specie va evidenziato:

a) che al di là delle ovvie tensioni prodotte anche nei figli da una separazione a dir poco controversa, non emergeva un quadro in cui i minori siano stati dolosamente coinvolti dal genitore in dinamiche violente, aggressive o prevaricazione, tanto da gettare gli stessi in una condizione di prostrazione e sofferenza psicologica per il clima di violenza e sopraffazione direttamente vissuto in casa;

b) che, al riguardo, è sufficiente ricordare, da un lato, che la perizia M.T. ha si rilevato comportamenti denigratori del signor T. nei confronti della madre dei bambini, ma ha al tempo stesso dato atto dell'assenza di anomalie o devianze nel comportamento tenuto dal padre nei confronti dei minori, tanto da proporre inizialmente il regime di affidamento condiviso, regime che peraltro, dalla "replica della CTU alle note di parte causa $S . / T$. “, risulta che inizialmente non fosse neppure osteggiato dalla S., che perseguiva l'obiettivo di trovare accordi per una condivisione della genitorialità dall'altro lato, che anche recentemente, pur dopo i fatti di (OMISSIS), il giudice civile, con provvedimento del 18.2.2014, non ha modificato il regime di affidamento condiviso dei figli per quanto riguarda direttamente la persona del T. , avendo unicamente imposto che questi non lasci i figli da soli con la Canestrari, attuale sua convivente;

c) che non basta ad integrare il delitto in addebito, il fatto che i figli siano stati in qualche occasione testimoni della condotta svalutante e denigratoria tenuta dal T. verso la moglie, non risultando che la condizione dei minori sia mai stata oggetto di allerta da parte dei servizi sociali per condotte direttamente o indirettamente maltrattanti tenute ai loro danni dal padre;

d) che, pertanto, il provvedimento impugnato va annullato limitatamente al capo $A$, con esclusivo riferimento ai maltrattamenti nei confronti dei figli, con conseguente cessazione della misura cautelare per quanto riguarda il divieto di avvicinamento ai figli e ai luoghi frequentati dai figli e di comunicazione con i figli medesimi.

5. Il P.M., nella sua impugnazione, prospetta il vizio di motivazione sotto $i$ profili della manifesta illogicità e contraddittorietà:

a) nella parte in cui, dopo aver affermato, con compendiosa, analitica, puntuale ed esaustiva motivazione, la sussistenza dei maltrattamenti in danno della moglie S.A., madre dei minori in argomento (reato abituale comprovato almeno a far data dall'anno 2010 fino all'aprile 2014 e successivamente)) conclude poi irragionevolmente per l'infondatezza della medesima illiceità in danno dei figli, e ciò, pur in un quadro di atti di violenza fisica e verbale direttamente esercitati nei confronti dei figli minori (negati peraltro dal Tribunale);

b) nella parte in cui, esclusa la violenza "diretta” Xil provvedimento ha sottovalutato l'efficacia maltrattante della "violenza assistita" dai figli che, al contrario, andrebbe pesata come idonea a cagionare un grave e duraturo stato di sofferenza in capo ai minori stessi;

c) nell'ulteriore parte che ignora che tale "violenza assistita", prima frutto della elaborazione della scienza psicologica e medica, poi della stessa elaborazione giurisprudenziale e quindi positivizzata con l'aggravante di cui all'art. 61 n. 1 quinquies c.p. (introdotta con la legge 119/13 del 15.10.13 Conversione in legge, con modificazioni, del decreto-legge 14 agosto 2013, n. 93, recante disposizioni urgenti in materia di sicurezza e per il contrasto della violenza di genere): trattasi invero di un concetto che richiama le sicure conseguenze negative, spesso indelebili, e le sofferenze patite dai minori quando, nel consorzio familiare di appartenenza, un genitore commetta maltrattamenti in danno dell'al- 
tro genitore, per la naturale sofferenza del minore nell'assistere ad atti di reiterata violenza fisica e/o verbale contro il genitore direttamente vittima della condotta maltrattante.

6. Il ricorso della parte pubblica prosegue poi con una serie di considerazioni teoriche sul tema rilevando:

a) che quando il fatto aggressivo non è isolato, ma duraturo nel tempo e caratterizzato dalla reiterazione abituale delle condotte maltrattanti, le sofferenze patite dai minori, ripetute nel tempo, integrano l'evento del delitto di maltrattamenti in danno dei minori medesimi, costretti ad assistere, vedere, sentire, percepire la condotta fisicamente, verbalmente, psicologicamente violenta in danno della madre;

b) che la prova di tali sofferenze è "dato notorio" emergente dalle discipline che studiano il bambino, non essendo controvertibile l'insorgenza di tale disagio e di tale dolore nelle comunità familiari caratterizzate da violenza domestica di un genitore nei confronti dell'altro;

c) che, diversamente opinando, non si comprenderebbe perché il legislatore abbia considerato necessario introdurre una aggravante di carattere generale, applicabile non solo al delitto abituale ma anche ai delitti contro la vita e l'incolumità individuale, caratterizzati da condotte uni sussistenti, tenuto comunque conto che ciò che la norma sanziona è il pregiudizio, cagionato al minore (esposto a violenza commessa in danno di terzi): conclusione questa particolarmente valida quando il destinatario delle violenze è la madre, per la considerazione della intensità e gravità delle sofferenze che tale condotta arreca al minore figlio.

\section{Considerato in diritto}

1. Ritiene questa Corte, interpretando una regola ermeneutica (espressa in un ben diverso caso di maltrattamento di persona disabile, ad opera di una badante convivente: Cass. pen. sez. 6, 9724/2013 Rv. 254472), che possano integrare il delitto di cui all'art. 572 cod. pen. non solo fatti commissivi, sistematicamente lesivi della personalità della persona offesa, ma anche condotte omissive connotate da una deliberata indifferenza e trascuratezza verso gli elementari bisogni affettivi ed esistenziali della "persona debole" da tutelare.

1.1. Regola questa che consente, nell'ambito della disamina della condotta maltrattante di un coniuge nei confronti dell'altro coniuge, di comprendere nel novero dell'offensività, tipica della norma, anche la "posizione passiva dei figli minori" laddove questi siano "sistematici spettatori obbligati" delle manifestazioni di violenza, anche psicologica (nella specie del padre nei confronti della madre).

1.2. Deve però trattarsi di un quadro di fatti commissivi, abitualmente lesivi della personalità materna, ma al tempo stesso connotati, in capo al soggetto maltrattante, e per la parte corrispondente alla "prole-presente", da "indifferenza omissiva", frutto di una deli- berata e consapevole insofferenza e trascuratezza verso gli elementari ed insopprimibili bisogni affettivi ed esistenziali dei figli stessi, nonché realizzati in violazione dell'art. 147 cod. civ., in punto di educazione e istruzione al rispetto delle regole minimali del vivere civile, cui non si sottrae la comunità familiare regolata dall'art. 30 della Carta costituzionale.

1.3. In altre parole, tale complessa realtà relazionale, per assumere rilievo nella sua dinamica patologica ed avere possibilità di positivo inquadramento nello schema dogmatico dell'art. 572 cod. pen., esige, oltre alla ricorrenza dell'elemento soggettivo, una necessaria iterazione e persistenza nel tempo, qualità queste apprezzabili in concreto dal giudice di merito, il quale solo, nella piena libertà di valutazione degli elementi di fatto, ha titolo per accertare $i$ termini, oggettivi e soggettivi, del comportamento incriminato, con specifico e puntuale riferimento alle singole interazioni familiari ed agli esiti negati$v i$, verificabili, nei processi di crescita morale e sociale della prole interessata, a tanto non bastando una mera generalizzata negatività della condotta in sé e per sé considerata.

1.4. Ciò premesso, questa Corte rileva che di tali parametri ha tenuto conto la gravata sentenza, la quale, prendendo atto che io stato di sofferenza e di umiliazione delle vittime non deve necessariamente collegarsi a specifici comportamenti vessatori posti in essere nei confronti di un determinato soggetto passivo, ha del pari correttamente argomentato sulla non comparabilità dei fatti che hanno determinato le decisioni della V e VI sezione di questa Corte (41142/2010 Rv. 248904; 8592/2010 Rv. 246028) rispetto all'odierna fattispecie, che si differenzia e si caratterizza appunto dalla "occasionante".

1.5. Da ciò consegue che il ricorso del P.M., pur apprezzabile nelle premesse in diritto sulla "violenza assistita" (rectius: "percepita"), ed in linea con le tendenze normative in atto (vds.: art. 1 d.l. 93/2013 convertito con legge 5 ottobre 2013 n.119), non è accoglibile in punto di fatto, avuto riguardo alla concreta motivazione del giudice di merito -espressa senza illogicità od incongruenze apprezzabili in sede di legittimità - la quale ha escluso:

a) che il padre abbia realizzato forme di maltrattamento diretto nei confronti della prole;

b) che la materialità della condotta dell'imputato, quale espressa nei confronti della madre dei minori, abbia assunto (come già detto) connotazioni diverse dalla "occasionante", le volte in cui $i$ figli erano testimoni-spettatori dei comportamenti illeciti del padre.

2. Il ricorso pertanto risulta infondato, valutata la conformità del provvedimento alle norme stabilite, nonché apprezzata la tenuta logica e coerenza strutturale della giustificazione che è stata formulata.

$$
\text { P.Q.M. }
$$

Rigetta il ricorso. 


\section{Terza Sentenza}

CASS. PEN. SEZ. III, SENT., (UD. 17-05-2016)

27-10-2016, N. 45403

\section{MALTRATTAMENTI IN FAMIGLIA \\ VIOLENZA SESSUALE}

In genere

\section{REPUBBLICA ITALIANA \\ IN NOME DEL POPOLO ITALIANO \\ LA CORTE SUPREMA DI CASSAZIONE \\ SEZIONE TERZA PENALE}

Composta dagli Ill.mi Sigg.ri Magistrati:

Dott. ROSI Elisabetta - Presidente -

Dott. DE MASI Oronzo - Consigliere -

Dott. MOCCI Mauro - Consigliere -

Dott. LIBERATI Giovanni - rel. Consigliere -

Dott. GAI Emanuela - Consigliere -

ha pronunciato la seguente:

\section{SENTENZA}

Sul ricorso proposto da:

P.A., nata a (OMISSIS) il (OMISSIS), quale tutore del K.G., nata a (OMISSIS) il (OMISSIS);

parte civile nel procedimento nei confronti di:

S.K., nato in (OMISSIS) il (OMISSIS);

- avverso la sentenza del 31/3/2015 del Giudice dell'udienza preliminare del Tribunale di Latina;

- visti gli atti, il provvedimento impugnato e il ricorso;

- udita la relazione svolta dal Consigliere Dott. LIBERATI Giovanni;

- udito il Pubblico Ministero, in persona del Sostituto Procuratore Generale Dott. ANGELILLIS Ciro, che ha concluso chiedendo l'annullamento con rinvio al Tribunale di Latina per nuovo giudizio;

- udito per la parte civile l'avv. RONCHINI, che ha concluso chiedendo l'accoglimento del ricorso;

- udito per l'imputato l'avv. DAVOLI Vincenzo, in sostituzione dell'avv. GALLO Rossella, che ha concluso chiedendo il rigetto del ricorso.

\section{Svolgimento del processo}

1. Con sentenza del 31 marzo 2015 il Giudice dell'udienza preliminare del Tribunale di Latina ha dichiarato non doversi procedere nei confronti di S.K. per il reato di cui all'art. 61 c.p., $n .11$ quinquies, art. 609 bis c.p., art. 609 ter c.p., $n .5$ ter (per avere costretto con violenza la convivente K.K. a subire un rapporto sessuale completo, costringendo la figlia minore K.G. ad assistere alla violenza), perchè il fatto non sussiste.

Ha ritenuto il giudice insufficienti gli elementi probatori raccolti per disporre il rinvio a giudizio dell'imputato, non essendo riscontrata la iniziale versione accusatoria della parte offesa da elementi certi, nè testimoniali nè documentali, alla luce delle ritrattazione della persona offesa innanzi al Pubblico Ministero e nel corso dell'incidente probatorio.

2. Avverso tale sentenza ha proposto ricorso la parte civile K.G., in persona del tutore, mediante il suo difensore, affidato ad un unico articolato motivo, mediante il quale ha prospettato erronea applicazione di legge in relazione al giudizio di inidoneità degli elementi acquisiti a sostenere l'accusa in giudizio, ai sensi dell'art. 425 c.p.p., e vizio di motivazione.

Ha escluso, in particolare, che la valutazione del giudice dell'udienza preliminare possa estendersi, qualora l'accusa sia fondata su prove dichiarative, alla valutazione di attendibilità dei dichiaranti, come invece avvenuto nella sentenza impugnata, in ragione della natura esclusivamente processuale della valutazione rimessa al giudice dell'udienza preliminare.

\section{Motivi della decisione}

Il ricorso è fondato

1. Va premesso che il ricorso, proposto dall'Avvocato P.A., quale tutore della minore K.G., nata il (OMISSIS), persona offesa costituita parte civile, è ammissibile, stante la piena legittimazione della minore, per effetto della contestazione della circostanza aggravante di cui all'art. 61 c.p., $n .11$ quinquies, che consente di ritenerla persona offesa.

1.1. Tale disposizione è stata introdotta dal D.L. 14 agosto 2013, n. 93, art. 1, comma 1, convertito con modificazioni nella L. 15 ottobre 2013, n. 119, e prevede un aggravamento di pena nei delitti non colposi contro la vita e l'incolumità individuale, contro la libertà personale e nel delitto di cui all'art. 572 c.p., quando il fatto sia commesso in presenza o in danno di un minore degli anni diciotto o in danno di una persona in stato di gravidanza.

In tal modo il legislatore ha inteso attribuire specifica valenza giuridica alla c.d. "violenza assistita", intesa come il complesso di ricadute di tipo comportamentale, psicologico, fisico, sociale e cognitivo, nel breve e lungo termine, sui minori costretti ad assistere ad episodi di violenza e, soprattutto, a quelli di cui è vittima la madre.

La disposizione $\dot{e}$, infatti, volta, nell'ambito del rafforzamento della tutela delle vittime di violenze domestiche, o, più in generale, di reati contro l'incolumità individuale e la libertà personale, a sanzionare, attraverso l'aggravamento del trattamento punitivo, l'esposizione del minore alla percezione di atti di violenza, sia nei confronti di altri componenti del nucleo familiare, sia di terzi, tra l'altro attuando una specifica indicazione contenuta in tal senso nell'art. 46 d) della Convenzione di Istanbul del 
Consiglio d'Europa sulla prevenzione e la lotta contro la violenza nei confronti delle donne e la violenza domestica.

1.2. Ne consegue, proprio in considerazione della ratio ispiratrice della disposizione e della sua funzione, che il minore che abbia assistito ad uno dei delitti indicati nella disposizione può essere considerato anch'egli persona offesa del reato, in quanto la configurabilità di detta circostanza aggravante determina una estensione dell'ambito della tutela penale, anche al minore che abbia assistito alla violenza, come tale pienamente legittimato a costituirsi parte civile, essendo anch'egli danneggiato dal reato, così come aggravato.

Ciò, d'altra parte, era già stato affermato dalla giurisprudenza di questa Corte in relazione al reato di maltrattamenti in famiglia, riconoscendo che integra il delitto di cui all'art. 572 c.p. anche l'esposizione del minore alla percezione di atti di violenza condotti nei confronti di altri componenti del nucleo familiare (v. ad es. Sez. 5, n. 41142 del 22 ottobre 2010, C., Rv. 248904 e Sez. 6, n. 8592/10 del 21 dicembre 2009, Z. e altri, Rv. 246028).

Ne deriva la piena legittimazione della minore, quale persona offesa, per effetto della contestazione di detta aggravante, a costituirsi parte civile nel procedimento relativo alla violenza sessuale commessa nei confronti della madre ed alla quale dovette assistere, e, dunque, a proporre ricorso avverso la decisione di proscioglimento dell'imputato.

2. Ciò premesso, giova ricordare che, secondo il consolidato orientamento interpretativo di questa Corte il giudice dell'udienza preliminare può pronunciare sentenza di non luogo a provvedere, ai sensi dell'art. 425 c.p.p., comma 3, solo quando il materiale probatorio sia assolutamente inidoneo a sostenere l'accusa in giudizio e cioè quando manchino le condizioni per una prognosi favorevole all'accusa: il giudizio, quindi, deve essere di mera valutazione processuale e non un vero e proprio giudizio di merito sulla colpevolezza dell'imputato, che compete solo al giudice del dibattimento (Sez. 2, n. 48831 del 14/11/2013, Maida, Rv. 257645; conf. Sez. 2, n. 46145 del 05/11/2015, Caputo, Rv. 265246; Sez. 5, $n$. 26756 del 26/02/2016, Miglietta, Rv. 267189; Sez. 2, n. 15942 del 07/04/2016, I., Rv. 266443).

Il giudice dell'udienza preliminare ha, dunque, il potere di pronunciare la sentenza di non luogo a procedere, ai sensi dell'art. 425 c.p.p., comma 3, solo quando l'insufficienza e la contraddittorietà degli elementi acquisiti rivestano caratteristiche tali da non poter essere ragionevolmente superabili nel giudizio (Sez. 6, n. 10849 del 12/01/2012, Petramala, Rv. 252280), anche tenendo conto della suscettibilità del compendio probatorio a subire mutamenti nella fase dibattimentale (Sez. 6, $n$. 29156 del 03/06/2015, Arvonio, Rv. 264053).

3. Ora, nella vicenda in esame, il Giudice dell'udienza preliminare, ha disposto il rinvio a giudizio dell'imputato S.K. in relazione ai reati di lesioni aggravate e mal- trattamenti di cui ai capi b) et c) della rubrica, sulla base di quanto esposto dalla persona offesa K.K. nella denuncia querela (nonostante le ritrattazioni innanzi al Pubblico Ministero e nel corso dell'incidente probatorio), nonchè di quanto dichiarato dalla minore K.G. al Pubblico Ministero (nonostante la sua ritrattazione nel corso dell'incidente probatorio) ed anche delle altre sommarie informazioni acquisite, prosciogliendo, invero contraddittoriamente, l'imputato dal reato di violenza sessuale commesso in danno della medesima K.K. (aggravato, ai sensi dell'art. 61 c.p., n. 11 quinquies, per avere costretto ad assistervi la figlia minore K.G., nata il (OMISSIS)), in considerazione della insufficienza degli elementi probatori raccolti per disporre il rinvio a giudizio.

Ha evidenziato, in particolare, il Giudice dell'udienza preliminare che la, peraltro iniziale, versione accusatoria della persona offesa, K.K., non era avvalorata da riscontri certi, nè documentali nè testimoniali, e che l'unica persona che aveva riferito, de relato, della violen$z a$, K.H., la aveva collocata cronologicamente al (OMISSIS) anzichè al (OMISSIS), sottolineando le ritrattazioni della persona offesa innanzi al Pubblico Ministero e nel corso dell'incidente probatorio.

4. Risultano evidenti, dunque, sia la insufficienza e la contraddittorietà della motivazione, che si limita a richiamare gli atti di indagine, senza indicarne, nemmeno in sintesi, il contenuto, e non consente, dunque, di verificare la correttezza del percorso logico seguito dal primo giudice, che tra l'altro ha, contraddittoriamente, ritenuto sufficienti le dichiarazioni della persona offesa per disporre il rinvio a giudizio dell'imputato in relazione agli altri reati contestati; sia, in ogni caso, l'esorbitanza dalle attribuzioni proprie del giudice dell'udienza preliminare, avendo, nella specie, il giudice, pur affermando l'insufficienza degli elementi raccolti per disporre il rinvio a giudizio dell'imputato, compiuto un vero e proprio giudizio sulla colpevolezza dell'imputato, tra l'altro senza tener conto adeguatamente della esistenza di significativi elementi a carico (costituiti dalle iniziali dichiarazioni della vittima K.K. e di K.H.) e dei loro possibili sviluppi nel corso del dibattimento, attraverso l'esame della persona offesa ed anche della minore.

Deve, dunque, ritenersi sussistente la violazione di legge denunciata dalla ricorrente, che comporta l'annullamento della sentenza impugnata e la restituzione degli atti al Tribunale di Latina.

\section{P.Q.M.}

Annulla la sentenza impugnata senza rinvio e dispone trasmettersi gli atti al Tribunale di Latina.

In caso di diffusione del presente provvedimento si omettano le generalità e gli altri identificativi a norma del D.Lgs. n. 196 del 2003, art. 52 in quanto imposto dalla legge.

Così deciso in Roma, il 17 maggio 2016.

Depositato in Cancelleria il 27 ottobre 2016 


\section{Quarta Sentenza}

Cass. pen. Sez. I, Sent., (ud. 02-03-2017) 14-03-2017, n. 12328

\section{INGIURIA E DIFFAMAZIONE MALTRATTAMENTI IN FAMIGLIA}

\section{REPUBBLICA ITALIANA \\ IN NOME DEL POPOLO ITALIANO \\ LA CORTE SUPREMA DI CASSAZIONE \\ SEZIONE PRIMA PENALE}

Composta dagli Ill.mi Sigg.ri Magistrati:

Dott. DI TOMASSI Maria Stefania - Presidente -

Dott. SIANI Vincenzo - Consigliere -

Dott. TALERICO Palma - Consigliere -

Dott. APRILE Stefano - rel. Consigliere -

Dott. MINCHELLA Antonio - Consigliere -

ha pronunciato la seguente:

\section{SENTENZA}

sul ricorso proposto da:

G.V., nato a (OMISSIS);

- avverso la sentenza del 27 gennaio 2016 pronunciata dalla Corte di assise di appello di Torino;

- visti gli atti, il provvedimento denunziato, il ricorso;

- udita la relazione svolta dal Consigliere Dott. Stefano Aprile;

- sentite le richieste del Pubblico Ministero, in persona del Sostituto Procuratore generale Dott. Ciro Angelillis, che ha concluso per il rigetto del ricorso;

- dato atto dell'assenza dei difensori delle parti civili;

- udito il difensore Avv. Raffaele D'Antino, che ha concluso per l'annullamento della sentenza.

\section{Svolgimento del processo}

1. Con il provvedimento impugnato, la Corte di assise d'appello di Torino ha confermato la sentenza del 22 dicembre 2014 del Giudice dell'udienza preliminare del Tribunale di Cuneo che, in sede di giudizio abbreviato, aveva dichiarato G.V. responsabile del delitto di omicidio aggravato della convivente A.S. (artt. 575 e 577 c.p., art. 61 c.p., comma 1, n. 11-quinquies), esclusa l'aggravante dei futili motivi, concesse le circostanze attenuanti generiche equivalenti rispetto all'aggravante, irrogando la pena di sedici anni di reclusione.

La Corte di merito, dopo avere fatto richiamo alla sentenza di primo grado per quello che concerne la non contestata responsabilità del ricorrente per l'omicidio della convivente cagionato mediante l'esplosione a distanza ravvicinata di tre colpi di arma da fuoco diretti al torace della vittima - colpi esplosi con l'arma della quale G. poteva legittimamente disporre in quanto
Guardia Particolare Giurata -, ha rigettato i motivi di appello riguardanti la sussistenza dell 'aggravante di cui all'art. 61 c.p., comma 2, n. 11-quinquies, e il trattamento sanzionatorio con riguardo al giudizio di equivalenza tra le circostanze attenuanti generiche e l'indicata circostanza aggravante.

2. Ricorre G.V., a mezzo del difensore avv. Raffaele D'Antino, che chiede l'annullamento della sentenza impugnata, formulando due motivi di ricorso, sostanzialmente riproducenti i motivi di appello.

2.1. Osserva, con il primo motivo, che la sentenza è nulla per l'inosservanza o erronea applicazione della legge penale o di altre norme giuridiche di cui si deve tener conto nell'applicazione della legge penale, a norma dell'art. 606 c.p.p., comma 1, lett. b), con riferimento all'art. 61 c.p., comma 1, n. 11quinquies, nonchè per la mancanza, contraddittorietà o manifesta illogicità della motivazione, a norma dell'art. 606 c.p.p., comma 1, lett. e), con riguardo alla citata aggravante che sarebbe stata ritenuta sussistente nonostante i figli minori, pur presenti nell'appartamento e nelle relative pertinen$z e$ in cui è avvenuto il fatto, non hanno allo stesso direttamente assistito.

2.2. Osserva, con il secondo motivo, che la sentenza è nulla per la mancanza, contraddittorietà o manifesta illogicità della motivazione, a norma dell'art. 606 c.p.p., comma 1, lett. e), con riguardo all'operato giudizio di bilanciamento, dovendosi ritenere la prevalenza delle concesse circostanze attenuanti generiche rispetto alla ritenuta aggravante, evidenziando la contraddittorietà della motivazione in relazione agli indici di cui all'art. 133 c.p., nonchè la contraddittorietà della motivazione derivante dalla ritenuta sussistenza delle circostanze attenuanti generiche che avrebbe dovuto indurre la Corte a operare un giudizio di prevalenza e, infine, la mancanza di motivazione in ordine alla determinazione della pena essendosi il giudice di merito discostato dal minimo edittale.

\section{Motivi della decisione}

1. Osserva il Collegio che il ricorso appare infondato.

1.1. Con riguardo al primo motivo di ricorso, deve essere ricordato che la L. 15 ottobre 2013, n. 119, che ha convertito, con modificazioni, il D.L. 14 agosto 2013, $n$. 93, ha provveduto a configurare una nuova aggravante comune, collocata nell'art. 61 c.p., comma 1, $n$. 11-quinquies, per il caso che i delitti non colposi contro la vita e l'incolumità individuale, contro la libertà personale, nonchè il delitto di maltrattamenti vengano commessi "in presenza o in danno di un minore di anni diciotto ovvero in danno di persona in stato di gravidanza", nel contempo eliminando la previsione originariamente inserita dal decreto-legge che aveva esteso l'aggravante speciale prevista dall'art. 572 c.p., comma 2, per il caso che il delitto di maltrattamenti in famiglia venga consumato ai danni di minori infraquattordicenni 
anche all'ipotesi in cui il reato sia commesso "alla presenza di un minore di anni diciotto".

In tal modo il legislatore non ha solo esteso l'ambito di applicazione delle speciali aggravanti previste dal decreto-legge per il delitto di maltrattamenti in famiglia, nonchè introdotto una nuova aggravante comune (quella dello stato di gravidanza della vittima del reato), ma ha altresi ampliato l'estensione di quella originariamente prevista dall'art. 572 c.p., comma 2, (reato commesso in danno di minore infraquattordicenne), attribuendo effetto aggravante al fatto commesso in danno non solo del minore infraquattordicen$n e$, bensì del minore degli anni diciotto (sulla continuità normativa si veda: Sez. 1, Sentenza n. 52181 del 8/11/2016, Brandi, non massimata).

1.2. Tanto premesso, è necessario interrogarsi sul significato della locuzione "in presenza (...) di un minore di anni diciotto", allo scopo di chiarire se sia richiesto che il fatto sia commesso davanti agli occhi del minore, come si sostiene nel ricorso, o sia sufficiente che lo stesso ne abbia comunque percezione e consapevolezza.

In proposito deve, da subito, sgombrarsi il campo dalla suggestione derivante dai primi commenti al decreto-legge che aveva previsto l'aggravante in discorso per il solo delitto di maltrattamenti in famiglia.

Si era, infatti, opinato che il legislatore dell'urgenza avesse inteso attribuire specifica valenza giuridica alla c.d. violenza assistita, intesa come il complesso di ricadute di tipo comportamentale, psicologico, fisico, sociale e cognitivo, nel breve e lungo termine, sui minori costretti ad assistere a episodi di violenza domestica e soprattutto a quelli di cui è vittima la madre.

Si era, inoltre, ritenuto necessario, stante la natura abituale del reato di maltrattamenti in famiglia, che i minori assistano a una pluralità di atti di maltrattamento.

In realtà, la giurisprudenza di legittimità aveva da tempo riconosciuto che integra il delitto di cui all'art. 572 c.p. anche l'esposizione del minore alla percezione di atti di violenza condotti nei confronti di altri componenti del nucleo familiare (Sez. $5, \mathrm{n}$. 41142 del 22 ottobre 2010, C., Rv. 248904; Sez. 6, $n$. 8592/10 del 21 dicembre 2009, Z. e altri, Rv. 246028), tanto che l'iniziale previsione del decretolegge deve essere apparsa allo stesso legislatore scarsamente innovativa del tessuto normativo di riferimento al punto da modificarla radicalmente in sede di conversione nella attuale previsione dell'art. 61 c.p., comma 1, n. 11-quinquies.

1.3. Da queste prime considerazioni può agevolmente desumersi, contrariamente a quanto sostenuto nel ricorso, che la presenza del minore, alla stregua del nuovo art. 61 c.p., comma 1, n. 11-quinquies, non riguarda la percezione di un comportamento abituale o reiterato, essendo sufficiente che lo stesso perce- pisca la condotta penalmente sanzionata dalla disposizione incriminatrice di parte speciale attinente i delitti contro la vita, l'incolumità personale e la libertà individuale.

1.4. La questione centrale è, però, quella attinente il concetto di commissione del fatto "in presenza (...) di un minore", con specifico riguardo alle modalità di percezione di esso da parte del minore.

Deve, cioè, essere chiarito se il concetto di "in presenza" coincida con quello di "al cospetto", sia dal punto di vista materiale (condotta posta in essere al cospetto e dunque davanti agli occhi del minore), sia dal punto di vista soggettivo (consapevolezza da parte dell'autore che il fatto è commesso in presenza del minore).

Per definire il concetto di "in presenza" è utile esaminare altre fattispecie di parte speciale che contengono la medesima proposizione.

1.4.1. Viene alla mente, innanzitutto, il delitto di cui all'art. 609-quinquies c.p., (corruzione di minorenni) il quale punisce "chiunque compie atti sessuali in presenza di persona minore di anni quattordici, al fine di farla assistere".

La giurisprudenza è orientata, in proposito, a ritenere che la specifica finalità di fare assistere il minore introduca un elemento attinente il dolo specifico (Sez. 3, Sentenza n. 12537 del 29/01/2015, R., Rv. 263000), giungendo a escluderne la sussistenza in presenza dell'apparente assopimento del minore (Sez. 3, Sentenza $n$. 15633 del 12/03/2008, M., Rv. 240035), mentre la "presenza" costituisce un elemento di fatto attinente la percepibilità dell'atto, non soltanto con il senso della vista.

1.4.2. Allo stesso modo, il delitto di ingiuria, già previsto dall'art. 594 c.p., richiedeva la commissione del fatto in presenza dell'offeso.

La giurisprudenza di legittimità era stabilmente orientata a ritenere che "per la configurabilità del delitto di ingiuria e sufficiente che la persona offesa, anche se non sia vista dal soggetto agente, abbia la possibilità di percepire ed effettivamente percepisca le espressioni ingiuriose" (Sez. 5, Sentenza n. 11909 del 04/07/1975, Alfano, Rv. 131429).

1.5. Si può, dunque, trarre una prima conclusione sul punto: per ritenere sussistente la circostanza aggravante in discorso è sufficiente che il minore percepisca il reato, non essendo richiesto che lo stesso sia commesso davanti ai suoi occhi.

Dal punto di vista dell'elemento psicologico, trattandosi di una circostanza aggravante di tipo oggettivo riguardante la modalità dell'azione a norma dell'art. 70 c.p., la stessa è valutata a carico dell'agente se conosciuta ovvero se ignorata per colpa o ritenuta inesistente per errore determinato da colpa, a norma dell'art. 59 c.p..

Ad avviso del Collegio, quindi, la circostanza aggra- 
vante di cui all'art. 61 c.p., comma 1, n. 11-quinquies, introdotta dalla L. n. 119 del 2013, è configurabile tutte le volte che il minore degli anni diciotto percepisca la commissione del reato e anche quando la sua presenza non sia visibile dall'autore il quale, tuttavia, ne abbia la consapevolezza o avrebbe dovuto averla usando l'ordinaria diligenza.

2. Essendosi, così, risolta la problematica concernente l'interpretazione del termine di "in presenza" contenuto nella circostanza aggravante di cui all'art. 61 c.p., comma 1, n. 11-quinquies, può agevolmente essere esaminato il primo motivo di ricorso.

Il motivo è del tutto infondato poiché la Corte d'assise di appello di Torino, e prima il GUP del Tribunale di Cuneo, hanno correttamente interpretato e applicato la norma di legge nel senso ritenuto da questo Collegio, dando, inoltre, ampia e logica motivazione delle conclusioni assunte in proposito, con accertamento di fatto incensurabile in questa sede, della accertata presenza del figlio minore A. nel soggiorno attiguo e comunicante, mediante un'ampia porta rimasta aperta, con il locale cucina ove è avvenuto l'omicidio, nonché dell'effettiva percezione del fatto da parte del minore che, oltre a piangere e urlare non appena compreso cosa era accaduto, riferiva alla vicina accorsa in aiuto che il padre aveva sparato alla madre.

Analoga coerente motivazione è stata stesa dalla Corte di merito con riguardo alla presenza e percezione del fatto da parte dell'altro figlio minore Giuseppe che si trovava nel giardino pertinenziale e che, uditi gli spari, era immediatamente accorso tanto che il ricorrente ha dichiarato di avere "cercato di chiudere la porta a soffietto per non fare vedere ai bambini" la madre morta.

3. Il secondo motivo di ricorso propone censure nel merito al percorso valutativo della Corte di assise di appello in punto di quantificazione della pena e di giudizio di bilanciamento, peraltro identiche a quelle già proposte in appello e alle quali si era già data ampia risposta nel relativo grado di giudizio, e dunque del tutto inammissibili.

La Corte di merito, con motivazione ampia, congruente, logica e non contraddittoria, ha esposto gli elementi in forza dei quali ha esercitato i propri poteri di quantificazione della pena e di comparazione delle circostanze attenuanti generiche con la ritenuta circostanza aggravante. $E^{\prime}$, in particolare, inammissibile perché risolventesi in censure su valutazioni di merito, insuscettibili, come tali, di aver seguito nel presente giudizio di legittimità, il motivo di ricorso concernente la misura della pena giacché la motivazione della impugnata sentenza, pure su tali punti conforme a quella del primo giudice, si sottrae a ogni sindacato per avere adeguatamente valorizzato la gravità della condotta omicida e delle precedenti azioni violente e minacciose elementi sicuramente rilevanti ai sensi dell'art. 133 c.p. - nonché per le connotazioni di complessiva coerenza dei suoi contenuti nell'apprezzamento della gravità dei fatti.

Il ricorrente indica elementi da considerare in senso posi- tivo (l'assenza di precedenti penali e l'esercizio dell'attività di Guardia Particolare Giurata), già esaminati e ampiamente valutati; in particolare, l'attività lavorativa, secondo la Corte di merito, avrebbe piuttosto dovuto spingere l'imputato a un maggiore autocontrollo e al rispetto delle regole del vivere civile e dei precetti penali.

Dall'ampia e coerente motivazione discende l'inammissibilità di qualsiasi censura o riserva in ordine ai criteri di scelta osservati dalla Corte di assise di appello, come del pari inammissibili risultano quelle che più specificatamente assumono la valutazione degli elementi all'uopo considerati risolvendosi, infatti, le stesse in censure in punto di fatto, insuscettibili, come tali, di aver seguito nel presente giudizio di legittimità, anche perché la motivazione della impugnata sentenza si sottrae a ogni sindacato in proposito per le connotazioni di coerenza, di completezza e di razionalità dei suoi contenuti.

4. Al rigetto del ricorso consegue, ai sensi dell'art. 616 c.p.p., la condanna del ricorrente al pagamento delle spese del procedimento.

$$
\text { P.Q.M. }
$$

Rigetta il ricorso e condanna il ricorrente al pagamento delle spese processuali.

Così deciso in Roma, il 2 marzo 2017.

Depositato in Cancelleria il 14 marzo 2017

\section{Quinta sentenza}

Cass. pen. Sez. VI, Sent., (ud. 18-10-2017) 14-12-2017, n. 55833

Fatto Diritto P.Q.M.

\section{MALTRATTAMENTI IN FAMIGLIA}

\section{REPUBBLICA ITALIANA \\ IN NOME DEL POPOLO ITALIANO \\ LA CORTE SUPREMA DI CASSAZIONE SEZIONE SESTA PENALE}

Composta dagli Ill.mi Sigg.ri Magistrati: Dott. PETRUZZELLIS Anna - Presidente Dott. TRONCI Andrea - Consigliere Dott. COSTANZO Angelo - Consigliere Dott. SCALIA Laura - Consigliere Dott. BASSI A. - rel. Consigliere -

ha pronunciato la seguente:

\section{SENTENZA}

sul ricorso proposto da:

V.C., nato il (OMISSIS) a (OMISSIS);

- avverso la sentenza del 20/01/2017 della Corte d'appello di Milano; 
- visti gli atti, il provvedimento impugnato e il ricorso;

- udita la relazione svolta dal Consigliere Dott. BASSI Alessandra;

- udito il Pubblico Ministero, in persona del Sostituto Procuratore Generale Dott. CANEVELLI Paolo, che ha concluso chiedendo che la sentenza sia annullata senza rinvio sull'aggravante dell'art. 61 c.p., comma 1, n. 11quinquies, e con rinvio ai fini della rideterminazione della pena.

\section{Svolgimento del processo}

1. Con il provvedimento in epigrafe, la Corte d'appello di Milano ha confermato la sentenza del 14 aprile 2016, con la quale il Giudice dell'udienza preliminare del Tribunale di Milano ha condannato, all'esito del giudizio abbreviato, V.C. alla pena di legge per i reati di maltrattamenti in danno della coniuge convivente, aggravato dalla presenza del figlio minorenne (capo $A$ ), e di lesioni personali aggravate in danno della medesima (capo B).

2. Avverso il provvedimento ha presentato ricorso l'Avv. Bora Luciano Maria, difensore di fiducia di V.C., e ne ha chiesto l'annullamento per i seguenti motivi:

2.1. violazione di legge penale in relazione al reato di cui al capo A), per avere la Corte d'appello ritenuto erroneamente integrato il delitto di maltrattamenti nonostante l'assenza del dolo (specifico e generico) e la mancanza dello stato di soggezione della vittima, là dove quest'ultima abbandonava l'abitazione coniugale dimostrando la mancanza di qualunque sudditanza psicologica nei confronti dell'imputato; il ricorrente si duole altresi della ritenuta integrazione della circostanza aggravante prevista dall'art. 61 c.p., comma 1, n. 11-quinquies, evidenziando che il minore, all'epoca dell'unico episodio in rilievo, aveva pochissimi mesi ed era totalmente incapace di comprendere cosa stesse accadendo, non potendo l'aggravante in parola correlarsi alla mera presenza fisica dell'infante al momento del fatto; il ricorrente si lamenta, infine, della mancata concessione delle circostanze attenuanti generiche;

2.2. violazione di legge penale in relazione al reato di cui al capo B), per avere la Corte erroneamente escluso l'assorbimento del reato di lesioni in quello di maltrattamenti.

\section{Motivi della decisione}

1. Il ricorso è destituito di fondamento in relazione a tutte le deduzioni mosse e va pertanto disatteso.

2. Non coglie nel segno il primo rilievo dedotto col primo motivo di ricorso.

2.1. Ed invero, la Corte milanese ha ineccepibilmente esplicitato le ragioni per le quali abbia ritenuto integrato il reato di maltrattamenti, evidenziando come, da un lato, risulti integrato lo specifico requisito della abitualità delle condotte, là dove le violenze si protraevano per ben sette mesi; dall'altro lato, sussista lo stato di soggezione della perso- na offesa, che il Collegio di merito ha ritenuto provato - con considerazioni scevre da illogicità manifesta - alla luce del comportamento altalenante della donna che, per mesi, non rivelava a nessuno le ripetute aggressioni e vessazioni subite dal marito, dopo la prima denuncia e l'adozione della misura cautelare dell'allontanamento dalla casa familiare, si riconciliava col coniuge, sporgeva, poi, un'ulteriore denuncia e perveniva, quindi, ad una nuova riappacificazione, a dimostrazione della condizione di inferiorità, sudditanza psicologica e timore nei confronti del marito (v. pagine 5 - 8 della sentenza impugnata).

3. E' inammissibile il rilievo concernente le circostanze attenuanti generiche là dove, secondo i consolidati principi espressi da questa Corte di legittimità, la concessione della diminuente costituisce un giudizio di fatto lasciato alla discrezionalità del giudice di merito, sottratto al controllo di legittimità qualora sia sorretto da una motivazione che - come nella specie - faccia emergere in misura sufficiente la sua valutazione circa l'adeguamento della pena alla gravità effettiva del reato ed alla personalità del reo (Sez. 6, n. 41365 del 28/10/2010, Straface, Rv. 248737; Sez. 3, n. 19639 del 27/01/2012, Gallo e altri, Rv. 252900).

La Corte territoriale ha congruamente argomentato come $V$. non sia meritevole dell'invocata mitigazione del trattamento sanzionatorio in assenza di situazioni e circostanze positive incidenti sull'apprezzamento dell'entità del reato e della capacità a delinquere dello stesso, valorizzando a conforto della conclusione la gravità della condotta e l'entità della violenza espressa (v. pagina 9 della sentenza impugnata).

4. E' destituito di fondamento anche il motivo con il quale il ricorrente attacca la decisione nella parte in cui si è ritenuta integrata la circostanza aggravante della presenza del minore di cui all'art. 61 c.p., comma 1, n. 11 quinquies, evidenziando come, all'epoca dell'unico episodio di rilievo, il figlio dell'imputato fosse troppo piccolo (avendo soltanto pochi mesi) per potersi rendere conto delle condotte maltrattanti in ipotesi commesse dal padre in danno della madre e, dunque, per subire le conseguenze negative cui appunto si correla l'aggravante in parola. Giova precisare che, secondo quanto si evince dal capo d'imputazione, il minore - nato nel maggio 2015 - aveva all'epoca dei vari episodi (commessi nel giugno, ottobre e novembre 2015) un'età compresa fra un mese sino a sei mesi.

4.1. L'art. 61 c.p.p., comma 1, n. 11-quinquies, come modificato con L. 15 ottobre 2013, n. 119 contempla una circostanza aggravante comune applicabile ai delitti non colposi contro la vita e l'incolumità individuale, contro la libertà personale, nonché - per quanto rileva nel caso sub iudice al delitto di maltrattamenti qualora la condotta sia commessa "in presenza o in danno di un minore di anni diciotto ovvero in danno di persona in stato di gravidanza”. 
4.2. La novellato del 2011 si pone su di una linea di continuità rispetto alla costante ermeneusi di legittimità, secondo la quale il delitto di maltrattamenti è configurabile anche nei confronti dei figli qualora la condotta di colui che compia atti di violenza fisica sia diretta contro la sola convivente, ma - appunto in presenza dei minori, integrando la c.d. "violenza assistita" o indiretta. Ai fini della integrazione della fattispecie di cui all'art. 572 cod. pen., lo stato di sofferenza e di umiliazione delle vittime non deve necessariamente collegarsi a specifici comportamenti vessatori posti in essere nei confronti di un determinato soggetto passivo, ma può derivare anche da un clima generalmente instaurato all'interno di una comunità in conseguenza di atti di sopraffazione indistintamente e variamente commessi a carico delle persone sottoposte al potere del soggetto attivo, i quali ne siano tutti consapevoli, a prescindere dall'entità numerica degli atti vessatori e dalla loro riferibilità ad uno qualsiasi dei soggetti passivi (Sez. 5, 22/10/2010 n. 41142, Rv. 248904; Sez. 6, 21/12/2009, n. 8592, Rv. 246028).

Va invero rimarcato come il reato di maltrattamenti sia un reato contro la famiglia (precisamente contro l'assistenza familiare) e come il suo oggetto giuridico sia costituito dai congiunti interessi dello Stato alla tutela della famiglia da comportamenti vessatori e violenti e dell'interesse delle persone facenti parte della famiglia alla difesa della propria incolumità fisica e psichica (ex plurimis Sez. 6, 24/11/2011 n. 24575, Rv. 252906). Coerentemente col bene protetto dall'incriminazione, il raggio di copertura della incriminazione non può non estendersi a comprendere tutti i soggetti che facciano parte della sfera familiare e che possano subire pregiudizi dai comportamenti aggressivi maturati in detto contesto.

D'altra parte, è consolidato l'orientamento della giurisprudenza civile secondo il quale i maltrattamenti commessi inflitti da un coniuge all'altro in presenza dei figli possono condurre alla dichiarazione di decadenza dalla potestà genitoriale, a norma dell'art. 330 cod. civ., per le inevitabili ripercussioni negative sull'equilibrio fisiopsichico della prole e sulla serenità dell'ambiente familiare e poichè, ancora, denotano mancanza di quel minimo di disponibilità affettiva e pedagogica richiesto in chi esercita la potestà parentale.

4.3. Lasciando da parte la previsione dell'aggravante dell'art. 61 c.p., comma 1, n. 11-quinquies, correlata all'agire che investa direttamente la persona del minore - che non interessa ai fini della soluzione sottoposto al vaglio della Corte -, si tratta di stabilire quale sia l'esatta portata della locuzione "in presenza (...) di un minore di anni diciotto". In particolare, per quanto concerne il caso de quo, v'è da chiedersi se, ai fini della integrazione dell'elemento circostanziale, sia sufficiente che il fatto sia commesso in un luogo ove si trovi contestualmente anche un minore ovvero se sia anche necessario che quest'ultimo sia in grado di percepire e di avere consapevolezza del carattere offensivo della condotta in danno di terzi avvenuta in sua presenza.

4.4. Orbene, ritiene il Collegio che, ai fini della integrazione della circostanza aggravante, sia sufficiente che il minore sia esposto alla percezione degli atti di violenza e che non sia in alcun modo richiesto che questi sia anche in grado, per il grado di maturità psicofisica conseguito, di realizzare, di comprendere la portata offensiva o lesiva degli atti commessi in sua presenza.

Tale conclusione esegetica discende dalla piana lettura della disposizione, interpretata secondo il senso fatto palese dal significato proprio delle parole secondo la connessione di esse - in conformità all'art. 12 preleggi -, là dove prevede esclusivamente che l'azione si svolga "in presenza" del minore e non richiede, in nessuna parte, che questi abbia raggiunto un'età o un grado di sviluppo intellettivo o psicologico tale da poter apprezzare la natura violenta o offensiva dell'agire che venga perpetrato intorno a sè.

Non potrebbe, pertanto, non risultare arbitraria l'introduzione per via interpretativa - giurisprudenziale - di una qualunque limitazione non prevista dal legislatore all'applicazione della fattispecie correlata all'età o al livello di maturità del soggetto che assista, suo malgrado, alle condotte vessatorie.

4.5. La lettura privilegiata si appalesa, d'altronde, coerente con la ratio dell'elemento circostanziale, che si correla all'esigenza di elevare la soglia di protezione di soggetti i quali, a cagione dell'incompletezza del loro sviluppo psico-fisico, risultano più vulnerabili e, dunque, più sensibili ed esposti ai riverberi negativi degli agiti aggressivi che siano realizzati in loro presenza.

Non è revocabile in dubbio il maggior disvalore della condotta maltrattante che sia posta in essere da un soggetto nei confronti di un altro in presenza di un minore, costretto ad essere suo malgrado spettatore delle manifestazioni di violenza, fisica o morale. Da un punto di vista oggettivo, l'azione aggressiva viene ad avere uno spettro più ampio, là dove va ad attingere una pluralità di soggetti anziché il solo partner, e realizza un'offesa di più grave intensità al bene tutelato dalla norma (la famiglia), comportando inevitabili ripercussioni negative nei processi $d i$ crescita morale e sociale della prole interessata; dal punto di vista dell'elemento soggettivo, manifesta una deliberata e consapevole trascuratezza dell'agente verso gli elementari bisogni affettivi ed esistenziali dei figli.

D'altronde, come condivisibilmente osservato dai Giudici della cognizione, costituisce approdo ormai consolidato della scienza psicologica che anche bambini molto piccoli, persino $i$ feti ancora nel grembo materno, siano in grado di percepire quanto 
avvenga nell'ambiente in cui si sviluppano e, dunque, di comprendere e di assorbire gli avvenimenti violenti che avvengano intorno a sè, in particolare le violenze subite dalla madre, con ferite psicologiche indelebili ed inevitabili riverberi negativi per lo sviluppo della loro personalità.

4.6. La lettura ermeneutica proposta è conforme all'insegnamento espresso da questa Corte in relazione ad altre ipotesi delittuose. In particolare, in materia di diffamazione, si è ritenuto integrato il requisito della comunicazione con più persone anche quando le frasi offensive siano pronunciate alla presenza di un adulto e di minori in tenera età (nella specie di due e quattro anni) qualora questi, pur non essendo in grado di cogliere lo specifico significato delle parole usate, ne abbiano colto la generica portata lesiva, tanto da esserne rimasti turbati e diventino potenziali strumenti di propagazione dei contenuti diffamatori (Sez. 5, n. $16108 \mathrm{del}$ 23/02/2017, L., Rv. 270672)

5. Non coglie nel segno neanche il secondo motivo di ricorso.

5.1. Il passaggio argomentativo col quale il Giudice d'appello ha ritenuto insussistenti i presupposti per l'invocato assorbimento nel delitto di maltrattamenti del reato di lesioni personali, di cui ha, fra l'altro, rimarcato l'estrema gravità (v. pagine 8 e 9 della sentenza in verifica), è invero perfettamente allineato al costante insegnamento di questa Corte, alla stregua del quale il reato di maltrattamenti in famiglia assorbe $i$ delitti di percosse e minacce, anche gravi, ma non quello di lesioni, attesa la diversa obiettività giuridica dei reati (Sez. 6, n. 13898 del 28/03/2012, S., Rv. 252585).

6. Dal rigetto del ricorso consegue la condanna del ricorrente al pagamento delle spese del procedimento.

$$
\text { P.Q.M. }
$$

Rigetta il ricorso e condanna il ricorrente al pagamento delle spese processuali.

Così deciso in Roma, il 18 ottobre 2017.

Depositato in Cancelleria il 14 dicembre 2017

\section{Sesta Sentenza}

\section{CORTE SUPREMA DI CASSAZIONE SEZIONE VI PENALE (ud. 23/02/2018) 02-05-2018, n. 18833}

Composta dagli Ill.mi Sigg.ri Magistrati: Dott. FIDELBO Giorgio - Presidente Dott. AGLIASTRO Mirella - Consigliere Dott. CRISCUOLO Anna - Consigliere Dott. BASSI Alessandra - rel. Consigliere Dott. SILVESTRI Pietro - Consigliere -

ha pronunciato la seguente:

\section{SENTENZA}

sul ricorso proposto da:

B.N., nata il (OMISSIS);

- avverso la sentenza del 04/04/2017 della Corte d'appello di Firenze;

- visti gli atti, il provvedimento impugnato e il ricorso;

- udita la relazione svolta dal Consigliere Dott.ssa BASSI Alessandra;

- udito il Pubblico Ministero, in persona del Sostituto Procuratore Generale Dott. ANIELLO Roberto, che ha concluso chiedendo che la sentenza sia annullata senza rinvio per prescrizione.

\section{Svolgimento del processo}

1. Con il provvedimento in epigrafe, la Corte d'appello di Firenze, in parziale riforma dell'appellata sentenza del Tribunale di Pistoia, ha dichiarato non doversi procedere nei confronti di B.N. in ordine ai reati di cui ai capi $B$ ), C) e D) (di calunnia, furto aggravato ed indebito utilizzo di bancomat) perchè estinti per sopravvenuta prescrizione ed ha rideterminato la pena alla medesima inflitta in ordine alla residua imputazione di cui al capo A), di maltrattamenti in danno dei due figli minori, commessa in concorso col convivente Be.Ma.

2. Nel ricorso a firma del difensore di fiducia Avv. **, B.N. chiede l'annullamento della sentenza per violazione di legge penale e vizio di motivazione in relazione alla ritenuta integrazione del reato di cui all'art. $572 \mathrm{cod}$. pen.. A sostegno del ricorso, evidenzia come, secondo la ricostruzione operata dei giudici della cognizione, i maltrattamenti sarebbero consistiti nell'aver costretto i figli minori a vivere in un clima di violenza, paura e continua tensione derivante dal fatto di dover assistere, quali spettatori passivi, alle violente dispute intercorse fra $i$ genitori coimputati, senza peraltro essere mai direttamente oggetto di aggressioni o soprusi né di violenza psicologica, come comprovato dal fatto che - giusta le conclusione del consulente tecnico del P.M. le presunte vittime non hanno manifestato alcun segno di disagio familiare.

\section{Motivi della decisione}

1. Il ricorso è fondato e la sentenza impugnata deve, pertanto, essere annullata per le ragioni di seguito esposte.

2. La decisione del ricorso ruota intorno alla configurabilità del delitto di cui all'art. 572 cod. pen. nel caso in cui la condotta in ipotesi maltrattante non si sia tradotta in comportamenti vessatori - fisici elo psicologici - rivolti direttamente verso la vittima, ma si sostanzi nel far assistere quest'ultima, quale spettatore passivo, alle condotte violente e offensive attuate nei confronti di altra persona. In particolare, avendo riguardo al caso sub iudice, se il reato di maltrattamenti in famiglia possa ritenersi integrato dalla condotta serbata dai genitori nei confronti dei loro figli minori (giusta contestazione) "per averli costretti a presenziare alle reiterate manifestazio- 
ni di reciproca conflittualità realizzate nell'ambito del rapporto di convivenza (....) mediante ripetuti episodi di aggressività fisica e psicologica, con condotte vessatorie e continui litigi, minacce e danneggiamenti di suppellettili, loro violente liti".

3. In risposta all'omologa deduzione mossa con l'atto d'appello, la Corte fiorentina ha argomentato che $i$ minori, sia pure non direttamente investiti dalle violenze e dai soprusi che hanno reciprocamente coinvolto la $B$. ed il coimputato, sono stati vittime di "violenza assistita", in quanto costretti dai genitori ad assistere passivamente alle loro feroci dispute, sebbene - come dato conto dal consulente tecnico del P.M. - essi non abbiano manifestato segnali di disagio familiare, circostanza - quest'ultima - stimata adeguatamente spiegabile con il periodo relativamente breve oggetto delle condotte contestate e con il fatto che "talune forme di imbarazzo" possono non essere state percepite dal consulente ( $v$. pagina 3 della sentenza impugnata).

4. In linea generale, mette conto di rilevare come il reato di maltrattamenti sia un reato contro la famiglia (precisamente contro l'assistenza familiare) e come il suo oggetto giuridico sia costituito dai congiunti interessi dello Stato alla tutela della famiglia da comportamenti vessatori e violenti e delle persone facenti parte della famiglia alla difesa della propria incolumità fisica e psichica (ex plurimis Sez. 6 del 24/11/2011 n. 24575, Rv. 252906).

4.1. In ossequio alla ratio ed al bene giuridico protetto, il raggio di copertura dell'incriminazione non può, pertanto, non estendersi a comprendere tutti i soggetti che facciano parte della sfera familiare e che possano subire un pregiudizio alla propria integrità psico-fisica a cagione dei comportamenti aggressivi maturati in detto contesto.

4.2. D'altra parte, va sottolineato come la norma all'art. 572 cod. pen. sanzioni la condotta di chi "maltratta", espressione verbale all'evidenza ampia (tanto da risultare, ad avviso di taluna dottrina, indeterminata), nell'ambito della quale possono pertanto rientrare non soltanto le percosse, le lesioni, le ingiurie, le minacce, le privazioni e le umiliazioni imposte alla vittima, ma anche gli atti di disprezzo e di offesa alla sua dignità, che si risolvano in vere e proprie sofferenze morali (Sez. 6, n. 44700 del 08/10/2013, P, Rv. 256962), potendo il reato essere difatti integrato anche mediante il compimento di atti che, di per sé, non costituiscono reato (Sez. 6, $n$. 13422 del 10/03/2016, O., Rv. 267270).

$\mathrm{Ne}$ discende che la condotta sanzionata dall'art. 572 cod. pen. non deve necessariamente collegarsi a specifici comportamenti vessatori posti in essere nei confronti di un determinato soggetto passivo, può realizzarsi tanto con un'azione, quanto con un'omissione, e può derivare anche da un clima generalmente instaurato all'interno di una comunità in conseguenza di atti di sopraffazione indistintamente e variamente commessi a carico delle persone sottopo- ste al potere del soggetto attivo (Sez. 5 del 22/10/2010 n. 41142, Rv. 248904; Sez. 6 del 21/12/2009, n. 8592, Rv. 246028).

4.3. Occorre rimarcare come l'ampiezza dei confini della materialità del reato sia stata "controbilanciata" in via interpretativa dalla duplice prescrizione che, da un lato, le condotte vessatorie siano state reiterate nel tempo (id est che sussista la c.d. abitualità); dall'altro lato, che l'agire criminoso sia connotato da idoneità offensiva rispetto al bene giuridico tutelato, e cioè che abbia cagionato uno stato di sofferenza psico-fisica nella vittima.

Su questa linea, questa Corte ha affermato che il delitto di maltrattamenti può essere integrato anche da comportamenti vessatori che si protraggano per un lasso di tempo limitato, a condizione che ciò sia utile alla realizzazione della ripetizione di atti vessatori idonea a determinare la sofferenza fisica o morale continuativa della parte offesa (Sez. 6, $n .1999 \mathrm{del}$ 09/12/1992 - dep. 1993, Gelati, Rv. 193273). Non è dunque necessario un comportamento vessatorio continuo ed ininterrotto perché il reato è caratterizzato da un'unità significante costituita da una condotta abituale che si estrinseca con più atti, delittuosi o no, che determinano sofferenze fisiche o morali, realizzati in momenti successivi ma collegati da un nesso di abitualità ed avvinti nel loro svolgimento da un'unica intenzione criminosa di ledere l'integrità fisica o il patrimonio morale del soggetto passivo: cioè, in sintesi, di infliggere abitualmente tali sofferenze (Sez. 6, n. 24727 del 27/04/2015, non massimata).

Deve dunque escludersi che la compromissione del bene protetto si verifichi in presenza di semplici fatti che ledono ovvero mettono in pericolo l'incolumità personale, la libertà o l'onore di una persona della famiglia, essendo necessario, per la configurabilità del reato, che tali fatti siano la componente di una più ampia ed unitaria condotta abituale, idonea ad imporre un regime di vita vessatorio, mortificante e insostenibile (in motivazione, la Corte ha precisato che fatti episodici lesivi di diritti fondamentali della persona, derivanti da situazioni contingenti e particolari, che possono verificarsi nei rapporti interpersonali di una convivenza familiare, non integrano il delitto di maltrattamenti, ma la propria autonomia di reati contro la persona) (Sez. 6, n. 27/05/2003, Caruso, Rv. 226794).

4.4. Sotto diverso aspetto, va notato come, secondo il consolidato orientamento della giurisprudenza civile, $i$ maltrattamenti inflitti da un coniuge all'altro in presenza dei figli possono condurre alla dichiarazione di decadenza dalla potestà genitoriale, a norma dell'art. 330 cod. civ., per le inevitabili ripercussioni negative sull'equilibrio fisiopsichico della prole $e$ sulla serenità dell'ambiente familiare e poiché, ancora, denotano mancanza di quel minimo di disponibilità affettiva e pedagogica richiesto in chi esercita la potestà parentale. 
4.5. Va, infine, rammentato come il reato di maltrattamenti richieda non un dolo intenzionale - inteso quale perseguimento dell'evento (sofferenza della vittima) come scopo finale dell'azione -, ma soltanto il dolo generico consistente nella coscienza e nella volontà di sottoporre la persona di famiglia ad un'abituale condizione di soggezione psicologica e di sofferenza (Sez. 6, n. 15680 del 28/03/2012, F., Rv. 252586).

5. Tanto premesso quanto agli elementi costituiti (oggettivo e soggettivo) del reato di cui all'art. $572 \mathrm{cod}$. pen., non è revocabile in dubbio che il delitto di maltrattamenti possa essere configurato anche nel caso in cui i comportamenti vessatori non siano rivolti direttamente in danno dei figli minori, ma li coinvolgano (solo) indirettamente quali involontari spettatori delle feroci liti e dei brutali scontri fra $i$ genitori che si svolgano all'interno delle mura domestiche, cioè allorquando essi siano vittime di c.d. violenza assistita. La condotta di chi costringa minore, suo malgrado, a presenziare - quale mero testimone - alle manifestazioni di violenza, fisica o morale, è certamente suscettibile di realizzare un'offesa al bene tutelato dalla norma (la famiglia), potendo comportare gravi ripercussioni negative nei processi di crescita morale e sociale della prole interessata.

D'altronde, costituisce approdo ormai consolidato della scienza psicologica che anche bambini molto piccoli, persino i feti ancora nel grembo materno, siano in grado di percepire quanto avvenga nell'ambiente in cui si sviluppano e, dunque, di comprendere e di assorbire gli avvenimenti violenti che ivi si svolgano, in particolare le violenze subite dalla madre, con ferite psicologiche indelebili ed inevitabili riverberi negativi per lo sviluppo della loro personalità.

5.1. Ritiene, nondimeno, il Collegio che il delitto di maltrattamenti scaturente da una condotta riportabile alla c.d. violenza assistita, proprio perché fondato su di una relazione non diretta, ma indiretta fra il comportamento dell'agente e la vittima - essendo l'azione rivolta a colpire non il minore, ma altri ovvero, come nella specie, connotandosi per la reciprocità delle offese fra i genitori - postula una prova rigorosa che l'agire - in ipotesi - illecito, per un verso, sia connotato dalla c.d. abitualità; per altro verso, sia idoneo ad offendere il bene giuridico protetto dall'incriminazione, id est abbia cagionato secondo un rapporto di causa-effetto - uno stato di sofferenza di natura psicofisica nei minori spettatori passivi.

5.2. Presupposti obbiettivi imprescindibili della condotta di cui la Corte non ha adeguatamente dato conto, là dove - con una motivazione all'evidenza sommaria ed assertiva - ha ripreso le conclusioni del consulente tecnico del P.M. secondo cui i bambini, pur non avendo manifestato alcun segno di disagio familiare, devono ritenersi "vittime di una forma di violenza assistita" e non ha verificato se effettivamente il rapporto estremamente conflittuale esistente fra i genitori, cui i figli erano costretti loro malgrado ad assistere, abbia avuto valenza maltrattante e tale da produrre la condizione di afflizione che connota il delitto.

6. Tanto premesso, la sentenza deve essere annullata per difetto di motivazione.

Nondimeno, il reato risulta medio tempore estinto per prescrizione, di tal che la decisione va rinviata al giudice civile per la pronuncia circa l'eventuale responsabilità civile scaturente dal delitto.

$$
\text { P.Q.M. }
$$

Annulla senza rinvio la sentenza impugnata perché il reato è estinto per prescrizione. Visto l'art. $578 \mathrm{cod}$. proc. pen. annulla medesima sentenza ai fin civili e rinvia per nuovo giudizio al giudice civile competente per valore in grado di appello.

Cosi deciso in Roma, il 23 febbraio 2018.

Depositato in Cancelleria il 2 maggio 2018

\section{Settima Sentenza}

\section{CASSAZIONE, SEZ. VI PENALE, SENTENZA 6 MARZO - 24 APRILE 2018, N. 18175}

\section{Presidente Fidelbo - Relatore Criscuolo}

\section{Ritenuto in fatto}

1. Il difensore di V.G. ha proposto ricorso avverso la sentenza indicata in epigrafe con la quale la Corte di appello di Palermo ha confermato la sentenza emessa in data 21 giugno 2016 dal Tribunale di Agrigento che aveva condannato l'imputato alla pena di anni 2 di reclusione per il delitto di maltrattamenti in danno della convivente, riconosciute attenuanti generiche equivalenti alla recidiva specifica reiterata infraquinquennale contestata.

Ne chiede l'annullamento per i seguenti motivi:

1.1. violazione dell'art. 8, comma 1, cod. proc. pen. in materia di competenza territoriale nonché contraddittorietà e manifesta illogicità della motivazione: deduce che la Corte di appello ha confermato la decisione del primo giudice, che aveva respinto l'eccezione di incompetenza territoriale sollevata, modificando la motivazione del giudice di primo grado, il quale aveva ritenuto che $i$ fatti oggetto di distinte denunce, la prima sporta a (...), la seconda a (...), fossero in continuazione e di pari gravità con conseguente radicamento della competenza nel luogo di commissione del primo reato ai sensi dell'art. 16 cod. proc. pen. mentre la Corte di appello ha giustificato la competenza del giudice di Agrigento erroneamente assimilando il reato abituale al reato permanente, al quale unicamente si applica il criterio di cui all'art. 8, comma 3, cod. proc. pen.. Il reato di maltrattamenti si consuma nel momento e nel luogo in cui le condotte in essere divengono complessivamente riconoscibili e qualificabili come maltratta- 
menti e nel caso di specie ciò è avvenuto in (...), dove fu sporta la denuncia per l'ultimo episodio del luglio 2010, mentre nella precedente denuncia la persona offesa riferiva un unico episodio, senza menzionare condotte precedenti di maltrattamento, cosicché prima della seconda denuncia non era emersa l'abitualità della condotta;

1.2. errata applicazione dell'art. 572 cod. pen. e vizio di motivazione, mancando nel caso in esame gli elementi tipici dl reato, in quanto manca l'abitualità della condotta e la persona offesa non è stata in grado di riferire alcun episodio specifico di ingiurie, minacce o violenza, limitandosi ad affermare che il compagno la picchiava spesso e la ingiuriava dicendole che gli aveva rovinato la vita; anche dopo la separazione, la riconciliazione e la nuova separazione non ha indicato fatti specifici, cosicché è stata in grado di circostanziare solo $i$ due episodi denunciati; manca lo stato di vessazione psicologica della vittima, non risultando che la persona offesa abbia vissuto in una condizione di soggezione o timore del compagno, tant'è che appena un mese dopo il primo allontanamento da casa, ritornò insieme al compagno a (...) e anche dopo la seconda denuncia condusse la figlia presso il padre;

1.3. violazione dell'art. 530, comma 1 e 2, cod. proc. pen. e vizio di motivazione, mancando la prova della responsabilità dell'imputato; è errata la valutazione delle dichiarazioni della persona offesa e della loro efficacia probatoria, non essendo la persona offesa attendibile per le lacune e contraddittorietà presenti nelle dichiarazioni rese in dibattimento, riportate nel ricorso, e per assenza di riscontri esterni, atteso che la madre della stessa non ha potuto riferire che un solo episodio né risultano episodi successivi a quello del 2009 né risultano acquisiti referti, annotazioni di p.g. o dichiarazioni testimoniali, mentre è stato trascurato l'unico documento prodotto dalla difesa, il verbale degli assistenti sociali presso il Tribunale per $i$ minorenni, ai quali la persona offesa dichiarò che la conflittualità tra i coniugi era alta e l'aggressività reciproca;

1.4. erronea applicazione degli artt. 581, 612 e 660 cod. pen. e mancanza di motivazione sulla richiesta riqualificazione dei fatti;

1.5. manifesta illogicità della motivazione in relazione al trattamento sanzionatorio, essendo stata applicata una pena di gran lunga superiore al minimo edittale previsto all'epoca dei fatti.

\section{Considerato in diritto}

1. Il ricorso è infondato per i motivi di seguito illustrati.

2. L'eccezione preliminare relativa alla competenza territoriale è infondata, nonostante risulti erroneo il criterio utilizzato dai giudici di appello.

A differenza del giudice di primo grado, che aveva ritenuto le due denunce sporte dalla persona offesa, la prima il 18 luglio 2009 a (...), la seconda il 14 luglio
2010 a (...), indicative di due episodi di maltrattamenti distinti ed in continuazione tra loro, con conseguente applicazione del criterio previsto dall'art. 16, comma 1, cod. proc. pen., la Corte di appello ha ritenuto erronea la valutazione in ragione della natura abituale del reato di maltrattamenti, caratterizzato da una serie di condotte verificatesi in un apprezzabile lasso di tempo, che isolatamente considerate potrebbero anche essere non punibili o non perseguibili, ma acquistano rilevanza penale per effetto della reiterazione nel tempo, cosicché le condotte devono essere considerate unitariamente e non sono scindibili né frazionabili in relazione al mutamento del luogo e al diverso contesto temporale in cui vengono poste in essere: pertanto, ha ritenuto applicabile la disciplina prevista per il reato permanente.

Pur muovendo da una premessa esatta, la Corte di appello è pervenuta ad un'errata conclusione, in quanto, se è vero che l'antigiuridicità delle condotte nel reato di maltrattamenti è correlata alla reiterazione di più atti lesivi dell'integrità fisica e morale della vittima ovvero da una serie di atti lesivi, in cui ogni singola azione è elemento della serie, al realizzarsi della quale si perfeziona il reato, ne discende che la struttura del reato è perdurante e continuativa, in quanto ogni azione si salda alla precedente, dando vita ad un reato unitario, definito "reato di durata", che mutua la disciplina della prescrizione dai reati permanenti, ma che si perfeziona con il compimento dell'ultimo atto della serie.

Questa Corte ha già avuto modo di affermare che è erronea l'equiparazione tra reato abituale e reato permanente al fine di individuare il luogo di consumazione del reato e quindi, il giudice competente, in quanto nel reato permanente l'azione è unica ed assume autonoma valenza antigiuridica fin dal primo atto della sua esecuzione, e protraendosi nel tempo, assume la qualità di condotta permanente, mentre nel reato abituale si è in presenza di singole condotte, da sole non idonee ad integrare quel determinato reato, che perdono la loro individualità come percosse, minacce, o quali condotte non rilevanti penalmente, per assumere la diversa configurazione giuridica per effetto della reiterazione. In tal caso è del tutto irrilevante giuridicamente individuare il momento iniziale della consumazione, in relazione ad una condotta di cui non può prevedersi l'inquadramento futuro, o improcedibile per mancanza di una condizione di punibilità, ma che assume rilevanza penale nella considerazione del comportamento complessivo. In tale ipotesi, il luogo del commesso reato ai fini della determinazione della competenza è quello in cui l'azione diviene complessivamente riconoscibile e qualificabile come maltrattamento, e si identifica nel luogo in cui la condotta viene consumata all'atto della presentazione della denuncia (Sez. 6, n. 52900 del 04/11/2016, Rv. 268559; Sez. 6, n. 43221 del 25/09/2013, Rv. 257461).

Conseguentemente, deve ritenersi corretta la valutazione del giudice di primo grado, atteso che, contrariamente alla prospettazione difensiva, il reato risulta contestato dal maggio 2009 al settembre 2010, dunque, da epoca 
precedente alla presentazione della prima denuncia, sporta nel luglio 2009, ed evidentemente riferita ad una serie di episodi pregressi, connotati da abitualità ed integranti il reato di maltrattamenti, come ritenuto dai giudici di primo grado e dimostrato dagli elementi valorizzati dai giudici di merito.

3. Anche nel merito il ricorso è infondato, ai limiti dell' $i$ nammissibilità, nella misura in cui ripropone pedissequamente, come si evince dalla lettura della sentenza impugnata, e con argomentazioni in fatto, le censure già formulate in appello, disattese dai giudici di secondo grado con motivazione puntuale, lineare e completa, che si salda a quella di primo grado, ancor più analitica, con la quale il ricorrente non si confronta.

4. Quanto all'attendibilità della persona offesa, concordemente ritenuta dai giudici di merito con apprezzamento in fatto, non censurabile in questa sede in assenza di incongruenze e di incoerenze valutative, è stato sottolineato che non vi è motivo di dubitare delle dichiarazioni rese dalla stessa, non essendo emerso alcun proposito vendicativo o gratuitamente calunniatorio della $G$. né la tendenza ad esasperare o esagerare nella narrazione delle liti e del regime di vita, progressivamente deterioratosi per le difficoltà economiche e per la dipendenza dal gioco del ricorrente.

Tale circostanza ha trovato conferma nelle dichiarazioni della madre della G., costretta ad inviarle periodicamente danaro, come ritenuto dai giudici di merito, $i$ quali hanno anche dato atto dell'attendibilità della testimone, avendo la stessa ammesso di non aver assistito a litigi o atti di violenza nei confronti della figlia, residente in Sicilia, ma di aver appreso dalla stessa della critica situazione e del mortificante regime di vita cui era sottoposta e di aver visto i segni sul corpo della figlia in occasione della prima separazione, quando, dopo aver sporto denuncia, la figlia si era trasferita con la bambina presso di lei.

L'episodio, puntualmente ricostruito in sentenza $e$ riscontrato dal referto medico, dà conto della gravità dello stesso, coinvolgente la figlia minore, e ben spiega la ragione della presentazione della denuncia, collocatasi al culmine di una serie di precedenti violenze, vessazioni e prevaricazioni, mai prima denunciate: a tal proposito, il giudice di primo grado ha dato atto che la persona offesa aveva riferito delle abituali minacce di morte rivoltele dal ricorrente, che minacciava anche di farle revocare la custodia della figlia, così da indurla ad evitare di sporgere denuncia.

Non rileva, contrariamente alla prospettazione del ricorrente, ai fini dell'attendibilità della persona offesa, il comportamento della persona offesa, determinatasi a riprendere la convivenza dopo poco tempo, nella speranza di un cambiamento dell'atteggiamento del compagno e di ricomporre il nucleo familiare, trattandosi di dinamiche frequenti e di motivazioni apprezzabili, che, tuttavia, non annullano le condotte maltrattanti precedenti, come ritenuto dai giudici di merito.

Tale argomentazione sostiene anche la valutazione del comportamento della persona offesa successivo alla nuova separazione ed al definitivo trasferimento presso la madre in Calabria: i giudici hanno infatti, rimarcato che le ragioni dell'allontanamento dalla casa familiare erano state ritenute valide anche dal Tribunale per $i$ minorenni, che aveva dichiarato non luogo a provvedere in ordine alla denuncia, sporta dal ricorrente nei confronti della compagna per sottrazione della minore nel febbraio 2010.

In relazione a tale periodo sono state sottolineate le condotte del ricorrente, trasferitosi in Calabria in un paese vicino a quello di residenza della compagna per controllarla, minacciarla, seguirla, appostandosi sotto casa e raggiungendola anche presso il luogo di lavoro, ove si verificava il litigio denunciato: in merito a tali condotte, di cui il ricorrente lamenta la mancanza di riscontri, la sentenza di primo grado dà conto delle dichiarazioni rese dallo stesso imputato circa le minacce telefoniche e degli elementi, che ne smentiscono la versione - v. pag. da 6 a 8 -.

Anche la rilevanza del contenuto del verbale degli assistenti sociali del Tribunale per i Minorenni di Palermo, asseritamente trascurata in sentenza, trova invece, puntuale valutazione nella sentenza di primo grado- pag. 6-, laddove si precisa che la persona offesa aveva riferito in dibattimento di essere stata istruita dal ricorrente sulle risposte da rendere anche con intimidazioni e minacce.

A fronte di tale ricostruzione analitica ed esaustiva risulta del tutto infondata la prospettata insussistenza del reato di maltrattamenti, dovendosi invece, ritenere corretta la valutazione dei giudici di merito, che hanno ravvisato nei fatti descritti la serialità e ripetitività degli atti lesivi descritti dalla persona offesa, tali da imporre un regime di vita mortificante, angosciante ed umiliante nonché di soggezione e timore nella persona offesa, indotto dalla gravità delle minacce di portarle via la figlia.

5. Manifestamente infondato è il dedotto vizio di motivazione in ordine alla richiesta derubricazione del reato nei reati di percosse, minaccia e molestia, disattesa dai giudici di merito con motivazione corretta ed ineccepibile in ragione della riconducibilità ad unità delle condotte in forza dell'abitualità dei comportamenti, non valutabili singolarmente ed isolatamente, bensì nella loro serialità, che li connota di ben maggiore gravità.

6. Generico è l'ultimo motivo, relativo al trattamento sanzionatorio, avendo i giudici giustificato la ritenuta congruità della pena inflitta, determinata in misura superiore al minimo edittale in ragione della pervicacia dimostrata dall'imputato nel seguire la persona offesa anche in Calabria nonché delle modalità e della non trascurabile durata della condotta, tenuto anche conto del benevolo riconoscimento di attenuanti generiche, equivalenti alla recidiva specifica reiterata infraquinquennale contestata. Al rigetto del ricorso consegue la condanna del ricorrente al pagamento delle spese processuali.

$$
\text { P.Q.M. }
$$

Rigetta il ricorso e condanna il ricorrente al pagamento delle spese processuali. 